\title{
Research on the optimization method for equipment allocation of chemical reconnaissance system based on the weighted Voronoi diagram
}

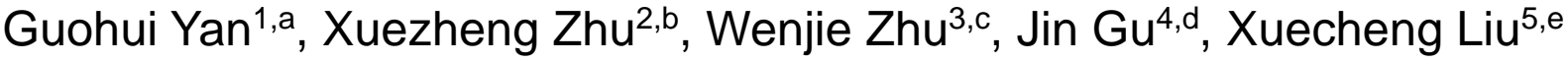 \\ Institute of NBC Defence, Beijing, 102205, China \\ aygh7678@sina.com
}

\begin{abstract}
Keywords: Chemical Reconnaissance; Equipment Application; Voronoi Diagram
\end{abstract}
\begin{abstract}
According to the maximum hollow circle strategy of the Voronoi diagram, Starting from the basic principles for equipment allocation of the chemical reconnaissance system, the capability range of the three kinds of chemical reconnaissance nodes is determined by the ideal model of chemical reconnaissance actions, and a optimization method for equipment allocation is established in the plane area which under the reconnaissance protection. The simulation experiment about the algorithm is carried out by taking a certain chemical reconnaissance protection as an example. Finally, the deficiencies of this method in theoretical significance and reality are analyzed, which provides a method and modeling foundation for the research on equipment application of chemical reconnaissance.
\end{abstract}

\section{Introduction}

The main task of chemical reconnaissance is to discover the symptoms of chemical attacks, identify the types of toxic agents, the infected cases and the secondary chemical hazards within the area of operations, as well as monitoring the spread and retention of the toxic agents clouds ${ }^{[1]}$, which provides the comprehensive and reliable chemical information for commanders to make decisions, and it's of great importance to guide chemical protection, exclude false alarms, eliminate chemical concerns, ensure the emotional stability of the troops, and make the operation intentions and actions without interference, so that it plays a very important role in the joint operations. In information operations, the professional equipment for chemical defense, the three-defense equipment of synthetic forces and the chemical reconnaissance equipment in main battle equipment need to be unified to set up a chemical reconnaissance system, so as to form the systematic chemical reconnaissance ability. In the system, all the equipment or devices involved in chemical reconnaissance are referred to as chemical reconnaissance nodes hereinafter. The limited chemical reconnaissance nodes should be used to ensure multiple important goals, whether the allocation is reasonable or not will directly affect the quality of the battle plan. Therefore, based on the theory of the weighted Voronoi diagram, this paper studied the optimization model for equipment allocation of the chemical reconnaissance system, which has a great significance for improving the scientificity of command and decision-making of the chemical reconnaissance, as well as for guaranteeing the correct and reasonable fighting-determinations from commanders.

\section{Brief Introduction about the Weighted Voronoi Diagram}

Voronoi diagram is a concept systematically established by the Russian mathematician G. Voronoi in 1908 and named after his name ${ }^{[2]}$, which is an important research content of computational geometry. With the concept of Voronoi diagram continuously penetrates into various application fields, it's widely used in the areas of graph and image processing, geographic information system, intelligent decision-making and the optimization algorithm ${ }^{[3]}$, especially the application and improvement of the weighted Voronoi diagram attracts more and more attentions.

The definition of the weighted Voronoi diagram is as follows. Suppose $P_{i}, i \in(1, n)$ are $\mathrm{n}$ points on the two-dimensional Euclidean space, $\lambda_{i}, i \in(1, n)$ are the given $\mathrm{n}$ positive real numbers. 


$$
V_{n}\left(P_{i}, \lambda_{i}\right)=I_{j \neq i}\left\{P \mid \frac{d\left(P, P_{i}\right)}{\lambda_{i}}<\frac{d\left(P, P_{j}\right)}{\lambda_{j}}\right\}
$$

Where $(i=1,2, \cdots n), V_{n}\left(P_{i}, j_{i}\right)$ divides the plane into $\mathrm{n}$ parts, the Plane segmentation determined by $V_{n}\left(P_{i}, \lambda_{i}\right)$ is called the weighted Voronoi diagram of point set, $\lambda_{i}$ is called the weight of $P_{i}$, the ratio of the two distances from any point in the field of the weighted Voronoi diagram to the generating elements of this field and from this point to the generating elements of adjacent area is less than the ratio of the two generating elements' weights.

When $\lambda_{1}=\lambda_{2}=\cdots=\lambda_{n}$, it is a standard Voronoi diagram, as shown in the left figure 1 below, It is a special case when all the generating elements' weights of the weighted Voronoi diagram of point set are equal. The following right figure 2 is a weighted Voronoi diagram.
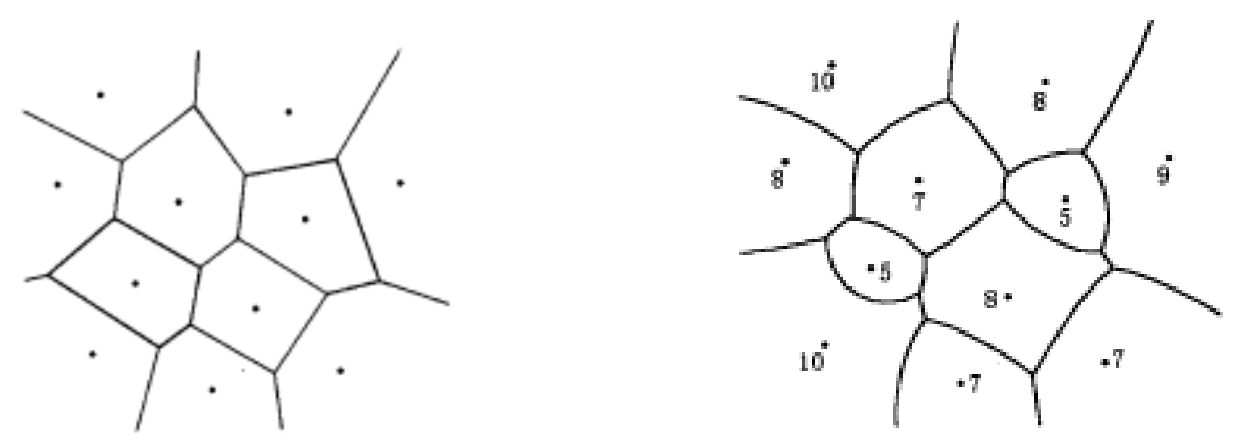

Figure 1. Example of the standard Voronoi diagram Figure 2. Example of the weighted Voronoi diagram

In the actions of chemical reconnaissance, different chemical reconnaissance nodes have different reconnaissance capabilities, which radiate outward form each node as the center form their respective capability ranges, finally the chemical reconnaissance network is set up, seeing from the definition and properties of the weighted Voronoi diagram, it is very suitable for the study of the optimal allocation for equipment of the chemical reconnaissance system.

\section{The optimization method for equipment allocation of the chemical reconnaissance system}

The primary task of chemical reconnaissance is to realize the first time perception in the event of chemical weapons attack or secondary chemical hazards, the ideal situation is that the chemical reconnaissance nodes can effectively cover the entire combat area, however, due to the large area of the battlefield, the demand for the number of nodes in allocation is too much, so the chemical reconnaissance nodes are limited combined with the reality. Therefore, only when the number of nodes can not meet the full allocation, optimizing the allocation of node position according to the significance of different locations can maximize the perception capability of the whole area.

\section{Problem Description.}

Suppose that the chemical reconnaissance protection is carried out in the region of an offensive operation, the nodes of the whole chemical reconnaissance system can be divided into three categories.

(1) The first class is a certain type of toxic agent warning facility installed on tank and troop crawler, which is allocated according to the needs of their operation actions. For the chemical reconnaissance system, such chemical reconnaissance nodes will not be allocated based on the needs of chemical reconnaissance, which can only be regarded as the random allocation nodes. Due to the width of primary cloud caused by the general chemical weapons attack will be greater than the distance between two tanks (or troop crawlers), which is assumed as $\mathrm{S}_{0}$, so the value of the capability range of such chemical reconnaissance nodes can be set as $\mathrm{S}_{0} / 2$.

(2) The second class is toxic agent warner from the three-defense group of synthetic forces, this kind of chemical reconnaissance nodes are similar to the first class, but they are basically allocated on a certain distance from the upwind direction of their units or branch units, which can be regarded as the nodes allocated according to a certain rule. 
(3) The third class is the professional equipment of chemical defense corps, namely a certain type of chemical and radiological reconnaissance vehicles. Such chemical reconnaissance nodes are allocated on the appropriate positions nearby the key targets, interconnecting and networking with the former two types of nodes, whose capability range is $* \mathrm{~km}$, which is assumed as $S_{1}$.

Through the above analysis we can see, issues about the optimal allocation for equipment of chemical reconnaissance system can be summed up as detecting the cavity size in the protection region under the condition of allocating the first kind of nodes at random and allocating the second kind of nodes regularly, then putting forward the allocation strategy of the third kind of nodes covering the higher priority areas ${ }^{[4]}$.

\section{Algorithm Description.}

According to the influence scope of the Voronoi diagram, the property of complete plane segmentation and the characteristic of maximum hollow circle, the corresponding maximum hollow circle of each Voronoi node should be the local maximum blank area in Voronoi diagram ${ }^{[5]}$, in theory, all those can become the growth point of the third kinds of chemical reconnaissance nodes. If the number of the new adding reconnaissance node is 1 , the center of each hollow circle in Voronoi diagram can be regarded as the locally optimal solution. While considering the center of the maximum hollow circle as the globally optimal solution, that is the growth point of the new reconnaissance nodes; if the number of new adding reconnaissance node is greater than 1, sorting the corresponding maximum hollow circle of each node in the Voronoi diagram according to its radius, the node with larger radius is the growth point of new reconnaissance nodes.

When the number of the growth point of the chemical reconnaissance node, which is calculated according to the maximum hollow circle strategy, is greater than 1, the relation between the distance of the two nodes' solutions $d_{i j}\left(q_{i}, q_{j}\right)$ and the capability range of this kind of nodes $\mathrm{S}_{1}$ should be considered, the threshold valuescan be determined by $\mathrm{S}_{1} / 2$ in general case.

The specific resolving steps are shown as follows:

(1) According to the allocation of the former two kinds of chemical reconnaissance nodes, the corresponding initial Voronoi diagram is generated;

(2) Solve the corresponding maximum hollow circle of each Voronoi polygon node $q_{i}$;

(3) Determine the value of eaccording to the capability range of the third kind of chemical reconnaissance nodes;

(4) Compare the distance $d_{i j}(i \neq j ; i, j=1,2,3 \ldots, n)$ between $q_{i}$ and $q_{j}$ of each node, if $d_{i j}<\varepsilon$, delete the corresponding node of the hollow circle with a smaller radius.

(5) Sorting the radius of the corresponding maximum hollow circles of the reserved nodes in a descending order, the ideal number $\mathrm{n}$ of the required and new adding third kinds of chemical reconnaissance nodes is obtained;

(6) Determine the location of nodes corresponding to the former $m$ hollow circles according to the number of new adding base stations $m(m<n)$, that is the location of the new adding third kinds of chemical reconnaissance nodes.

\section{Simulation Experiment}

Suppose that the protection area of a certain chemical reconnaissance is roughly a rectangle $* m \times * m, 200$ the first and the second kinds of chemical reconnaissance nodes have been deployed in the initial stage of operation, its reconnaissance capabilities range $S_{0}=* m$, the demand for the third kinds of chemical reconnaissance nodes can be calculated by simulation under the ideal condition. Assuming that the number of the third kinds of nodes can be added is 6 , selecting the allocation location, and weighting based on the generated. The initial Voronoi diagram is generated as shown in figure 3. From the 13 ideal points calculated based on the algorithm, the former 6 are selected as the allocation positions of the third kinds reconnaissance nodes, after the addition, the weighted Voronoi diagram is generated as shown in figure 4. 


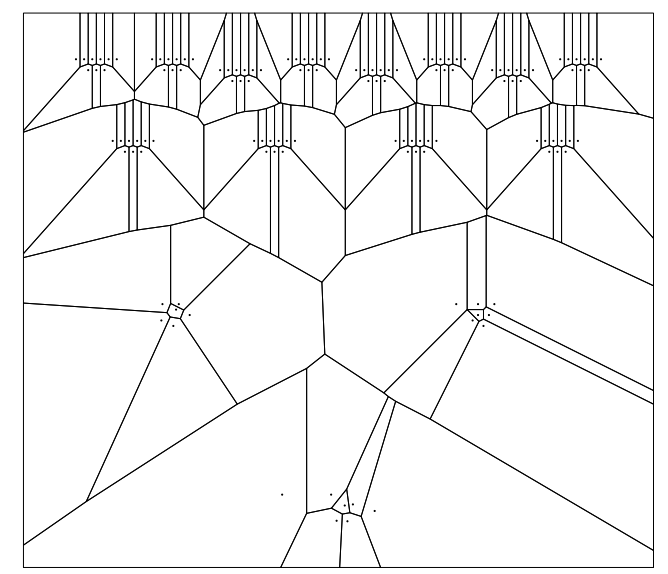

Figure 3. The Voronoi diagram of initial allocation

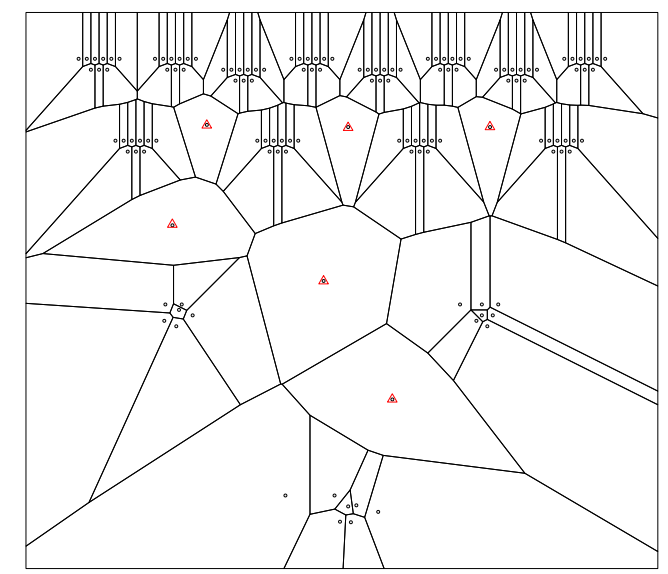

Figure 4. The weighted Voronoi diagram of optimal allocation eighted Voronoi diagram we can see, selecting the allocation location based on the algorithm can preferably meet the need of chemical reconnaissance protection.

\section{Conclusion}

The proposed allocation method of the chemical reconnaissance nodes are obtained based on the geometric properties of the Voronoi diagram under an ideal condition. So it must satisfy the following assumptions:

(1) The protection region must be an ideal plane area without interference.

(2) The first and the second class of nodes in initial allocation must possess the characteristics of omni-directivity and uniformity.

Based on the Voronoi diagram, the equipment allocation method of chemical reconnaissance system obtained by using the maximum hollow circle strategy can make the distribution of the third kinds of chemical reconnaissance nodes more reasonable and improve the coverage rate of chemical reconnaissance, which has a certain theoretical and guiding significance in the actual equipment allocation. Due to the influences from geography, interferences and equipment etc., in the actual equipment allocation, not only the assistances from GIS system and other professional databases but also the actual analysis on geographical environment and meteorological environment are both needed. According to the analysis results and combined with the actual test data, a more ideal allocation method can be achieved by the local and optimal adjustment on the positions of chemical reconnaissance nodes.

\section{Reference}

[1] Military Terminology Management Committee, Academy of Military Sciences. Military Terminology of the Chinese People's Liberation Army[M].Beijing: Military Science Press, 2011:842

[2] Yu Jia. Research on the base station selection method of the emergency communication system based on Voronoi diagram[J]. Information Communication, 2013,(2):35-37

[3] Xiaoyong Wu. Study on the optimization method for searching capability of the Anti-Submarine system [D].Changsha: National University of Defense Technology,2012:11

[4] Mahboubi H,Habibi J,Aghdam A G,et al. Distributed deployment strategies for improved coverage in a network of mobile sensors with prioritized sensing field. IEEE Transactions on Indusrial Informatics,2013,9(1):451-461

[5] Jun Liu etc. An optimization algorithm for node deployment of a reliable perception-oriented sensing network[J]. Journal Of Jilin University (Engineering Edition), 2015,45(6):1941-1945 